\title{
O projeto Research Domain Criteria e o abandono da tradição psicopatológica
}

\author{
Rafaela Zorzanelli*1 \\ Paulo Dalgalarrondo*2 \\ Cláudio E. M. Banzato*3
}

O artigo explora os pressupostos epistemológicos do Research Domain Criteria (RDoC) — projeto de investigação empírica dos transtornos mentais, dentro de uma plataforma biológica - promovido pelo National Institute of Mental Health (NIMH). Discute-se o ataque feito ao sistema DSM, pouco antes do lançamento de sua quinta versão, a partir da perspectiva neurocientífica adotada pelo RDoC. A aposta na patofisiologia, combinada com a exclusão da experiência descrita em primeira pessoa, aponta um cenário em que a pesquisa e a clínica se divorciam, com o risco do advento de uma estranha psiquiatria sem psique e sem pathos. Palavras-chave: RDoC, sistemas de classificação, transtornos mentais, DSM, clínica psiquiátrica

\footnotetext{
${ }^{*}$ Universidade do Estado do Rio de Janeiro - UERJ (Rio de Janeiro, RJ, Br)

*2 Universidade Estadual de Campinas - Unicamp (Campinas, SP, Br)

${ }^{* 3}$ Universidade Estadual de Campinas - Unicamp (Campinas, SP, Br)
} 


\section{O ataque ao DSM no contexto do projeto Research Domain Criteria}

No dia 29/4/2013, pouco antes do lançamento do DSM-V pela American Psychiatric Association (APA), um post intitulado Transforming Diagnosis foi publicado por Thomas Insel, diretor do National Institute of Mental Health (NIMH) dos EUA, em seu blog oficial do instituto (NIMH Director's Blog) (Insel, 2013), causando enorme repercussão pública e um indisfarçável mal-estar no interior da psiquiatria. Ao contrário da expectativa triunfante gerada por edições anteriores, a quinta versão do DSM foi apresentada de forma bastante anticlimática e recebida com ceticismo. Afinal, segundo Insel, as categorias diagnósticas do DSM, baseadas em conjuntos de sintomas clínicos e não em medidas laboratoriais objetivas (como em outras áreas da medicina), não teriam validade ${ }^{1}$ científica. É preciso esclarecer que o sentido de validade adotado por Insel é o de uma inscrição biológica definida, dada sua suposição de que: "mental disorders are biological disorders involving brain circuits that implicate specific domains of cognition, emotion, or behavior" (Insel, 2013). ${ }^{2}$

O post aplica uma verdadeira pá de cal nas pretensões clínicas e científicas do DSM com as afirmações taxativas de que "patients with mental disorders deserve better" e de que a busca de biomarcadores não será bem-sucedida enquanto as categorias do DSM forem consideradas o 'padrão ouro'. Preparado o terreno, Insel (2013) anuncia que o NIMH (principal agência de fomento científico dos EUA na área de psiquiatria) irá reorientar suas pesquisas se afastando do DSM. O pano de fundo desse ataque é constituído pela recente iniciativa, lançada pelo NIMH, do Research Domain Criteria (RDoC). Por ora, basta dizer que se trata

${ }^{1}$ Cumpre esclarecer que 'validade' é um termo muito usado nas discussões nosológicas em psiquiatria e parece ser o objetivo comum de todos, mas o que cada um entende por isso raramente é explicitado. Para uma discussão aprofundada da noção de 'validade' em psiquiatria consultar Rodrigues (2012).

${ }^{2}$ Reconhecendo o caráter algo dúbio dessa formulação, Rose (2013) diz que sua avaliação depende de como interpretamos palavras como 'are', 'involving' e 'implicate', se as tomamos como causas fundamentais ou se como elementos em vias biopsicossociais complexas e altamente individualizadas. 
de um projeto para transformar o diagnóstico, por meio da incorporação da genética, neuroimagem, ciência cognitiva, e outros níveis de informação com o fim de estabelecer as bases para um novo sistema classificatório (Insel, 2013). Segundo seus proponentes, o RDoC marcaria o início, na psiquiatria, da era da 'medicina de precisão' (nos moldes do que tem acontecido hoje no diagnóstico e tratamento individualizado do câncer - comparação recorrente na literatura psiquiátrica, a oncologia se tornou o novo modelo a ser emulado pela psiquiatria ${ }^{3}$ ). Por fim, o post conclui com uma citação de dois influentes pesquisadores em genética psiquiátrica conclamando-nos a mirar mais alto no que diz respeito ao diagnóstico psiquiátrico. Um desses autores, Nick Craddock, aliás, comenta em outro lugar sobre o paradoxo atual de possuirmos poderosas ferramentas do século XXI enquanto ainda nos digladiamos com conceitos e abordagens do século XIX (Casey et al., 2013).

Talvez esse seja um marco privilegiado do desencanto com o DSM e um acerto de contas com a realidade. Depois das grandes esperanças depositadas no sistema DSM por muitos pesquisadores (desde o DSM-III, de 1980) — de que seus recortes seriam confirmados empiricamente pela genética e pelas neurociências — teríamos a fria constatação de que tal promessa não se cumpriu. Curiosamente, para tais pesquisadores, o DSM deixou de ser considerado parte da solução para se tornar parte do problema. Suas categorias diagnósticas, que, segundo Steve Hyman (antecessor imediato de Thomas Insel na direção do NIMH), teriam a bizarra propriedade de serem, a um só tempo, muito amplas (na medida em que identificam populações notavelmente heterogêneas), e muito restritas (quem preenche critérios de um transtorno frequentemente também se qualifica para outros - comorbidade artefactual) e constituiriam um verdadeiro obstáculo ao progresso do conhecimento neurocientífico sobre os transtornos mentais (Casey et al., 2013). Isso aconteceria porque as investigações empíricas quase sempre partem, por variadas razões, ${ }^{4}$ dos recortes e distinções categoriais do DSM. Nas palavras de Insel (2012): "one reason we do not have biomarkers for mental disorders is our presumption that the biomarker is only valid if it maps on to a 'fictive category' rather than developing diagnostic categories based on the experimental data, as proposed by RDoC, our version of 'precision medicine'." Nessa perspectiva, o erro de origem seria a atribuição, inadvertente e implícita, de ‘significado biológico' a conjuntos de sintomas.

${ }^{3}$ Isso parece indicar um salto em termos da ambição propulsora: não se trata mais de tornar a psiquiatria apenas uma medicina regular, mas de equipará-la àquelas especialidades mais bem-sucedidas, que hoje se situam no front científico do conhecimento médico.

${ }^{4}$ Entre outras, contam-se a exigência de órgãos reguladores (por exemplo, o FDA exige que os ensaios clínicos para a aprovação de um novo medicamento utilizem as categorias do DSM), e as preferências editoriais de periódicos científicos, que priorizariam a publicações de estudos realizados de acordo com o referencial diagnóstico do DSM. 


\section{OBSERVANDO A PSIQUIATRIA}

Nos termos do pronunciamento conjunto de $13 / 5 / 2013$, assinado por Thomas Insel (NIMH) e Jeffrey Lieberman (APA), realizado para assegurar aos pacientes que o DSM e a CID ainda representam a melhor informação atualmente disponível para o diagnóstico clínico e tratamento dos transtornos mentais, lemos que o que é realisticamente factível para clínicos hoje não é mais suficiente para pesquisadores ${ }^{5}$ (Insel \& Lieberman, 2013). Em outros termos, o DSM passou de ícone da psiquiatria científica a uma ferramenta clínica provisória, que tem o inconveniente de obstruir o desenvolvimento científico. O que para alguns seria motivo de decepção, para outros seria apenas o tardio reconhecimento de que classificações diagnósticas são mapas performativos dos problemas enfrentados na realidade clínica. Existe uma ironia histórica nessa virada, pois o DSM sempre foi muito criticado por constranger e empobrecer a clínica psiquiátrica, mas esse era afinal o preço a pagar por uma psiquiatria científica no futuro. Com o DSM esvaziado de sua pretensão científica, a clínica, antes menosprezada, se transforma em sua principal razão de ser.

Em certo sentido, o projeto RDoC opera com pressupostos mais explícitos do que o sistema DSM. As cartas estão na mesa e sua aposta na patofisiologia é clara. Os mecanismos cerebrais, base do RDoC, sempre constituíram (ao menos implicitamente) parte essencial do horizonte de validação das categorias descritivas do DSM. ${ }^{6} \mathrm{O}$ raciocínio subjacente a esse movimento é o seguinte: se o território mapeado por tais categorias não coincide com aquele gerado pela progressiva identificação dos circuitos cerebrais, por que não tentar trilhar o caminho reverso? Isso supostamente garantiria que os recortes comportamentais gerados no final do processo teriam inscrição neurobiológica bem definida.

Diante da insatisfação crescente com o DSM, que teria falhado por não conseguir realizar, com suas sucessivas edições, a passagem do plano sintomatológico para o etiológico, ${ }^{7}$ o NIMH em seu Plano Estratégico de 2008 estabelece como seu

${ }^{5}$ Nesse pronunciamento de tom conciliador (embora de natureza retórica e política), afirma-se que o DSM e o RDoC são plataformas complementares e não rivais, na busca pelo progresso científico na caracterização de doenças e transtornos (Insel \& Lieberman, 2013).

${ }^{6}$ No caso dos transtornos mentais, as lesões cerebrais não são identificáveis, como acontece na neurologia, presume-se assim a existência de uma disfunção nos circuitos neurais (Insel et al., 2010). Não entraremos aqui na questão das fronteiras difusas e movediças entre ambas as especialidades médicas, com seus múltiplos determinantes. Interessa-nos, sobretudo, discutir a ambição de fazer da psiquiatria uma espécie de 'neurologia do mental e do comportamento'.

${ }^{7}$ No livro A Research Agenda for DSM-V, editado por Kupfer, First, e Regier, e publicado pela APA em 2002, é revelada logo na introdução, e com todas as letras, que era essa a meta perseguida: "the eventual development of an etiologically based, scientifically sound classification system" (p. XV). 
primeiro objetivo promover descobertas no cérebro e nas ciências comportamentais para impulsionar as pesquisas sobre as causas dos transtornos mentais (NIMH, 2008). Quatro estratégias são listadas para atingir tal objetivo, são elas: 1. Desenvolver um entendimento integrativo dos processos básicos cérebro-comportamento; 2. Identificar fatores genéticos e ambientais associados com os transtornos mentais; 3. Identificar e integrar os marcadores biológicos (biomarcadores) e os indicadores comportamentais associados com os transtornos mentais; 4. Desenvolver, para fins de pesquisa, novas formas de classificar os transtornos mentais baseadas em dimensões observáveis do comportamento e em medidas neurobiológicas (NIMH, 2008).

$\mathrm{O}$ projeto RDoC é concebido como parte dessa última estratégia, livrando as pesquisas das amarras do DSM. Sua matriz conceitual (construtos que representam supostos domínios de atividade e que refletiriam uma tipologia de funções e relações empíricas com atividades correlatas de circuitos cerebrais, examinados em diferentes unidades de análise) é completamente agnóstica em relação às categorias diagnósticas do DSM. É importante frisar que o RDoC não se opõe ao DSM, mas dele prescinde para sua existência e, no limite, pretende torná-lo obsoleto, atingindo os mesmos objetivos, mas com outros conceitos e por outros caminhos. Em vez de mais do mesmo (caso do DSM-V), o RDoC seria um game-changer, algo de que a psiquiatria necessitaria muito, segundo alguns autores (Casey et al., 2013).

Existe uma afirmação, quase sempre feita em tom que mistura lamento e esperança, que é recorrente na literatura psiquiátrica: a de que os diagnósticos psiquiátricos ainda são baseados somente nos relatos subjetivos dos pacientes e na observação, por parte do médico, do comportamento dos pacientes. A palavra 'ainda', nesse contexto, parece cumprir uma dupla função, indicar que essa situação atual é indesejável (mas não definitiva!), e que isso representa uma limitação a ser superada com o desenvolvimento científico da psiquiatria. Tipicamente, encontramos tal afirmação no início de textos que pretendem mostrar quais seriam os caminhos futuros da psiquiatria em sua busca de marcadores objetivos, que trariam uma confirmação independente e objetiva do diagnóstico psiquiátrico. O trecho a seguir, publicado numa revista de divulgação científica de grande prestígio, a Scientific American, pelo destacado neurocientista Steve Hyman (2003), fornece o exemplo acabado dessa posição: "in most branches of medicine, physicians can base their diagnoses on objective tests: a doctor can examine $\mathrm{x}$-rays to see if a bone is broken, for example, or extract tissue samples to search for cancer cells. But for some common and serious psychiatric disorders, diagnoses are still based entirely on the patient's own report of symptoms and the doctor's observations of the patient's behavior" (Hyman, 2003).

Entretanto, a posição inversa também tem seus defensores de peso no cenário psiquiátrico. Kenneth Kendler (2005), em artigo muito citado, publicado no American Journal of Psychiatry, estabelece como primeiro princípio da plataforma conceitual e filosófica que ele propõe, para a disciplina, que "psychiatry is irrevocably grounded 
in mental, first-person experiences". Para Kendler (2005), essa ancoragem no mundo mental nada tem de contingente: "foundational to this framework is the view that the field of psychiatry is deeply and irreversibly wedded to the mental world". Afinal, o objetivo da psiquiatria é o alívio do sofrimento humano que resultaria de alterações disfuncionais em certos domínios da experiência subjetiva, como humor, percepção, e cognição. A experiência, descrita na primeira pessoa, seria o ponto de partida da investigação clínica, conformando os eventuais alvos terapêuticos identificados. O trabalho clínico implicaria a avaliação e interpretação constantes da experiência subjetiva. Kendler, especialista em genética psiquiátrica e uma das mais respeitadas vozes da psiquiatria científica, não compartilha, em absoluto, da ideia de que os avanços neurocientíficos tornariam ociosa a dimensão experencial: "while we want to take advantage of the many advances in the neurosciences and molecular biology, this cannot be done at the expense of abandoning our grounding in the world of human mental suffering" (Kendler, 2005). Analisando a relação entre a psiquiatria e as neurociências, Serpa Jr. (2004) também rejeita o reducionismo eliminativista e mostra que é possível 'redescobrir' o cérebro sem eclipsar o sujeito.

Retornando ao RDoC, cumpre ressaltar que as controvérsias em torno desse projeto não se limitam de forma alguma à questão da busca por biomarcadores que conferissem maior objetividade ao diagnóstico psiquiátrico, expectativas de resto perfeitamente legítimas. O núcleo do debate é outro, menos relacionado aos objetivos do que aos pressupostos do RDoC. Como foi muito bem apontado por Berenbaum (2013), a plataforma RDoC parece pressupor que os fenômenos biológicos são de certo modo mais fundamentais que os fenômenos psicológicos para o conjunto dos transtornos mentais. ${ }^{8}$ Esse autor recusa tal primazia biológica com o argumento, que nos parece convincente, de que "a wide variety of etiological factors, both within and outside the individual, have been implicated in the etiology of psychopathology, and that there is no empirical or conceptual reason to privilege any particular level of explanation". Miller $\&$ Keller (2000), em artigo curto e inspirado (também citado por Berenbaum), fazem críticas conceituais certeiras ao reducionismo prevalente em diversos campos, como a psicologia, a medicina e as neurociências básicas e clínicas. Com muita elegância e precisão, eles desmistificam o erro que está na origem da posição reducionista: "it is not a property of biological data that they "underlie' psychological data" (Miller \& Keller, 2000). E acrescentam que uma dada teoria pode propor que esse seja o caso, mas aí teríamos uma proposta teórica e não um fato sobre os dados (Miller \& Keller,

${ }^{8}$ Sobre este ponto, deve-se considerar que quase todas as taxonomias psiquiátricas são muito heterogêneas, listando condições bastante variadas, nas quais os elementos mais fundamentais e os acessórios variam profundamente. 
2000). Em resposta a Berenbaum (2013), Cuthbert \& Kozak (2013) recusam a acusação de que o RDoC incorreria em um fundamentalismo biológico, mas reconhecem que a linguagem empregada pelos proponentes do projeto pode realmente dar essa impressão. Eles admitem a evidente ênfase na biologia, mas afirmam que o RDoC é sobretudo um esforço integrativo, que conta em sua matriz com múltiplas unidades de análise. Por fim, acrescentam que, embora o risco de reducionismo não possa ser eliminado, o RDoC não teria um caráter inerentemente reducionista.

\section{RDoC: Raízes da Escolha}

$\mathrm{O}$ RDoC assume claramente que o campo da psiquiatria necessita de um paradigma integralmente novo para a abordagem e investigação dos transtornos mentais. Em relação ao objeto de estudo da ciência psiquiátrica, com o qual deve ocupar-se a pesquisa no futuro próximo, tal paradigma assenta-se em três perspectivas básicas: antimentalismo, antissubjetivismo e certa forma de behaviorismo. Chamamos aqui de behaviorismo à determinada utilização da noção de comportamentos observáveis ou mensuráveis que está sendo proposta, em substituição aos sintomas subjetivos da tradição psicopatológica. Ao contrário da tradição behaviorista, na qual eles bebem grandes porções, mas porções selecionadas, os proponentes do RDoC não creem que o centro de estudo da ação humana é o comportamento, seus determinantes e consequências. Os propositores do RDoC creem que o epicentro do humano, normal e patológico, é o cérebro, os circuitos neuronais, células, receptores, sinalizadores e o controle genético a eles relacionados. Por isso, curiosamente sugerem um sistema baseado em ferramentas metodológicas do behaviorismo (sobretudo aquelas provindas da pesquisa animal experimental e da tradição psicométrica relacionada à pesquisa neurobiológica), mas recusam, sem assumir, os pressupostos fundamentais do behaviorismo.

Assim, em vez dos sintomas vivenciados, relatados na primeira pessoa, e dos comportamentos observáveis no contexto da clínica, bases da tradição psicopatológica - que de uma forma ou de outra aparecem, sobretudo, nos relatos das pessoas acometidas e de seus familiares - , o RDoC propõe que se baseie a pesquisa psiquiátrica, não mais em tal conjunto impreciso e subjetivo de dados, mas em comportamentos observáveis, seguros, marcadamente objetivos e não influenciados pela subjetividade das pessoas comuns. A experiência vivida por quem padece das síndromes psiquiátricas deve ser substituída por algo mais objetivo e supostamente mais científico.

$\mathrm{Na}$ frase de Insel \& Lieberman (2013), "it is increasingly evident that mental illness will be best understood as disorders of brain structure and function that implicate specific domains of cognition, emotion, and behavior". São transtornos da estrutura e função cerebrais que implicam o comportamento, este é somente o sinal 
resultante daquilo que realmente interessa. $\mathrm{O}$ novo sistema taxonômico a ser utilizado irá aproximar as amostras de comportamentos coletados em direção às abordagens da genética molecular e neurociências para, enfim, se compreender de modo plenamente científico as doenças ditas mentais.

A proposta do RDoC é que se dirijam os esforços para além de um sistema cujo eixo foi até agora o diagnóstico baseado em sintomas. Seria necessário assentar a pesquisa nos mecanismos das doenças e em elementos que se conectem mais clara e diretamente a tais mecanismos. Os sistemas atuais, DSM e CID, baseados em sintomas (e não em biomarcadores e eventos comportamentais observáveis, mensuráveis, manejáveis no contexto da pesquisa neurobiológica e genética) deveriam, portanto, ser abandonados, no campo da pesquisa psiquiátrica.

\section{Continuidade ou descontinuidade da Psicopatologia com a Psicologia}

Uma das críticas que os defensores do RDoC fazem ao sistema DSM é que este se limita a uma taxonomia de transtornos mentais, excluindo toda a gama de comportamentos normais e subsindrômicos, que seriam importantes para uma pesquisa ampla em neurociências comportamentais, e que dariam conta de tudo o que se estende do normal ao patológico, em vários espectros do comportamento humano.

Segundo Hyman (Belluck \& Carey, 2013), um dos equívocos importantes do DSM é que tal sistema delimita e escolhe um modelo no qual "all psychiatric illnesses were represented as categories discontinuous with "normal"'. Isto se relacionaria à incapacidade do sistema de enxergar processos unitários que estariam na base tanto de conjuntos de síndromes, em parte superpostas (catalogadas erroneamente como comorbidades), como de continuidades entre os fenômenos normais e os ditos patológicos.

Embora tenha se tornado algo básico, na tradição psicopatológica (vide os grandes tratados de psiquiatria), ordenar os elementos básicos da doença mental a partir das funções mentais (atenção, sensopercepção, memória, pensamento, linguagem, afetividade, vontade etc.), derivando ou articulando o patológico com o normal, foi com Karl Jaspers que uma separação clara e muitas vezes radical entre os estados e fenômenos normais da vida mental e alguns dos fenômenos psicopatológicos (pelo menos alguns dos mais centrais relacionados à psicose, como delírio e alucinação) passou a ser defendida por um número significativo de autores.

A questão da continuidade entre os estados e fenômenos mentais/comportamentais normais e os patológicos em contraposição à suposta originalidade e especificidade do patológico é algo que não foi esclarecido de forma empírica e minimamente consensual. Partir da noção de que as (ou algumas das) síndromes delimitadas pela psiquiatria são 
específicas, ou estão em continuidade com fenômenos normais, são duas abordagens sem fundamentação empírica suficiente.

\section{Possíveis consequências clínicas do RDoC}

O apontamento crítico de Hyman (Casey et al., 2013), segundo o qual as categorias do DSM padeceriam, a um só tempo, de amplitude e restrição poderia ser o golpe desejado por muitos na estrutura classificatória desse manual, gerando uma abertura para novas formulações que aplacassem as críticas ${ }^{9}$ que o DSM vem recebendo há algum tempo. Em especial a de ser pretensamente 'ateórico', mas fomentando, na prática, uma psiquiatria reducionista no sentido biológico. No entanto, a crítica enunciada pelo RDoC, não só ao DSM mas ao capítulo sobre transtornos mentais da CID, indica, ao contrário disso, um impulso ainda maior no estreitamento da psiquiatria em torno da pesquisa de mecanismos biológicos capazes de desvendar ou de estabilizar, no sentido fleckiano do termo, categorias psiquiátricas em uso. Teríamos assim, dentro dos DSMs, um deslocamento de sistemas theory-laden (até a terceira edição) para sistemas symptom-based, sendo que o RDoC romperia com essa tradição, adotando uma proposta centrada na investigação de mecanismos patofisiológicos específicos e na atribuição de suas assinaturas celulares, subcelulares e moleculares, dentro do espectro de fenótipos clínicos já reconhecidos.

Dessa forma, o RDoC já suscita, antes mesmo de sua utilização, uma fonte de controvérsias, assumindo o projeto de classificar transtornos mentais a partir da análise de genes, células, circuitos neurais e de, no limite, prometer mais validade e confiabilidade etiológica do que as classificações existentes (Sisti et al., 2013). A teoria da doença mental que o inspira é flagrantemente naturalista, deixando entrever que uma psicopatologia seria suficientemente realizada apenas por meio da investigação de mecanismos neurobiológicos deficitários que ocorreriam nos organismos. A premissa que o sustenta é de que transtornos mentais são tipos naturais, desvendáveis por tecnologias de ponta, que alçariam a psiquiatria ao status de outras especialidades

${ }^{9}$ Entre as demais críticas ao DSM, destacamos a de ser um manual que favorece falsos positivos, estendendo ao infinito o processo de patologização de comportamentos ditos normais; de manter fortes relações com a indústria farmacêutica, pela presença maciça de conflito de interesses entre os participantes das forças-tarefas responsáveis pela descrição dos transtornos; de retirar importância das narrativas dos pacientes em prol de check-lists de sintomas; e de não passar de uma etnopsiquiatria, que refletiria a visão de homem, de saúde e doença vigente em sociedades liberais urbanas no Ocidente, com pretensões de uma psiquiatria global. 
médicas, podendo prescindir de relatos subjetivos, que ainda mantêm a psiquiatria em território incerto e movediço.

É dentro desse cenário que as asserções de Georges Canguilhem (1943/2002) se mostram pertinentes e em consonância com uma crítica ao projeto de prescindir da subjetividade na pesquisa dos transtornos mentais. Parte de seus argumentos é erigida em contraste com as proposições do estatístico Adolphe Quêtelet, no intuito de mostrar que o que se considera normalidade biológica não pode ser definido por critérios estatísticos. O 'normal', em se tratando da experiência de tudo que é vivo, não diria respeito apenas ao pertencimento a uma faixa dentro da distribuição da curva de Gauss, mas a uma experiência judicativa.

Uma consequência fundamental da distinção da patologia como valor, e não como desvio da média estatística, é a concepção de que uma variação no organismo não é, necessariamente, uma doença. O patológico derivaria, prioritariamente, de um sentimento da vida contrariada, e nem toda diversidade física e mental traria esse desdobramento como consequência. Não havendo uma dimensão fenomenológica, experiencial e corporificada de determinada variação do organismo, geradora de sofrimento, ela será apenas variação, e não patologia. Mas se uma anomalia ou variação do corpo é experimentada negativamente, restringe-se a capacidade de ser normativo, será considerada patológica. Em poucas palavras, se a anomalia não apresenta repercussão para o indivíduo, ela será ignorada ou concebida como uma variedade indiferente. $\mathrm{O}$ que decidiria se uma anomalia seria variação ou patologia é a reação particular que aquele organismo estabelece com seu meio e sua experiência de sofrimento. $\mathrm{O}$ autor nos permite concluir que a descoberta do fato patológico não é tributária apenas, nem exclusivamente, da ciência, mas da denúncia humana de seu próprio mal-estar. É a queixa que julgaria o estado em que a vida se encontra, não o desvio da norma estatística.

A fisiologia pode oferecer identificações claras sobre doenças - não é esse o ponto de insurgência de Canguilhem. Seu argumento é o de que é essencialmente a clínica que chancela a doença e a reconhece, porque a experiência de sofrimento e de restrição da capacidade vital são condições prioritárias para nomear uma variação física ou mental de doença - o que só pode ocorrer a partir da experiência do relato em primeira pessoa. Não é sem motivo que o espaço da clínica impõe, de forma radical, o dilema tão conhecido da generalidade das categorias produzidas em tensão com o caráter único de cada pessoa que sofre.

O projeto de investir na assinatura biológica de transtornos mentais nos colocaria - em contrapartida a uma medicina baseada em valores onde a psiquiatria se apoiaria - diante de uma clínica sem experiência, ou de uma psiquiatria sem psique (Parnas, 2014), uma vez que o substrato patofisiológico de um transtorno mental é justamente aquilo de que não se pode ter uma experiência vivida. Desse modo, o sofrimento envolvido nos sintomas de um transtorno, os sinais de mal-estar, as restrições, o relato singular do paciente não seriam os elementos centrais em torno dos quais o 
desvendamento do transtorno mental seria investigado, mas sim obstáculos à plena realização do projeto epistemológico do RDoC.

Em suma, excluída de saída a experiência e a clínica, aposta-se que seria possível reencontrá-las, no futuro, a partir de inscrições biológicas determinísticas e específicas, com o logos não apenas precedendo o pathos, mas falando por ele. Não nos parece, todavia, que a experiência silenciada na origem possa ser recuperada a partir de dados neurobiológicos objetivos gerados a partir da perspectiva de terceira pessoa. Concluindo, com o RDoC corre-se o risco de se colocar as poderosas ferramentas do século XXI, potencialmente muito férteis, a serviço de preconceitos do século XIX.

\section{Referências}

Belluck, P. \& Carey, B. (2013). Psychiatry's Guide Is Out of Touch With Science, Experts Say. New York Times, May, 6. Disponível em: <http://www.nytimes.com/2013/05/07/ health/psychiatrys-new-guide-falls-short-experts-say.html?pagewanted=all\&_r=0> Acesso em: 16 ago. 2013.

Berenbaum, H. (2013). Classification and Psychopathology Research. Journal of Abnormal Psychology, 122,(3), 701-894.

Canguilhem, G. (2002). O normal e o patológico. Rio de Janeiro: Forense Universitária. (Trabalho original publicado em 1943).

Casey, B.J. et al. (2013, nov.) DSM-V and RDoC: progress in psychiatry research? Nature Reviews Neuroscience, 14, 810-814.

Cuthbert, B. N. \& Kozak, M. J. (2013). Constructing Constructs for Psychopathology: The NIMH Research Domain Criteria 2013. Journal of Abnormal Psychology, 122(3), 928-937.

Hyman, S. E. (2003, Sept.). Diagnosing Disorders. Scientific American, 97-103.

Insel, T. (2010, Jul). Research Domain Criteria (RDoC): Toward a New Classification Framework for Research on Mental Disorders. American Journal of Psychiatry, 167(7), 748-751.

Insel, T. (2012, Oct.). NIMH Director's Blog: Words Matter. 2 October 2012. Recuperado em 29 de março de 2014 de <http://www.nimh.nih.gov/about/director/2012/ words-matter.shtml>.

Insel, T. (2013, Apr.). NIMH Director's Blog: Transforming Diagnosis. 29 April 2013. Recuperado em 15 de março de 2014 de <http://www.nimh.nih.gov/about/director/2013/ transforming-diagnosis.shtml>.

Insel, T. \& Lieberman, J. (May, 2013). DSM-V and RDoC: Shared Interests. NIMH Press Release 13 May 2013. Recuperado em 15 de março de 2014 de <http://www.nimh.nih. gov/news/science-news/2013/dsm-5-and-rdoc-shared-interests.shtml $>$. 


\section{OBSERVANDO A PSIQUIATRIA}

Kendler, K. S. (2005, March). Toward a Philosophical Structure for Psychiatry. American Journal of Psychiatry, 162(3), 433-440.

Kupfer, D. J., First, M. B., \& Regier, D. A (Eds.). (2002). A Research Agenda for DSM-V. Washington, D.C.: American Psychiatric Association.

Miller, G. A. \& Keller, J. (2000, Dec.). Psychology and Neuroscience: Making Peace. Current Directions in Psychological Science, 9(6), 212-215.

National Institute of Mental Health. Strategic Plan. (2008). Recuperado em 12 de fevereiro de 2014 de <http://www.nimh.nih.gov/about/strategic-planning-reports/nimh-strategic-plan-2008.pdf $>$.

Parnas, J. (2014, Feb.). The RDoC program: psychiatry without psyche? World Psychiatry, 13(1), 46-47.

Rodrigues, A. C. T. (2012). O que é validade na nosologia psiquiátrica. Tese (Doutorado em Ciências Médicas). Universidade Estadual de Campinas - Unicamp. Recuperado em 2 de abril de 2014 de <http://www.bibliotecadigital.unicamp.br/ document $/$ ?code $=000845736>$

Rose, N. (2013, Jun.). What is Diagnosis For? Lecture given at the Institute of Psychiatry (London) on 4 June 2013. Recuperado em 15 de março de 2014 de $<$ http://nikolasrose. com/wp-content/uploads/2013/07/Rose-2013-What-is-diagnosis-for-IoP-revised-July-2013.pdf $>$.

Serpa Jr., O. D. de. (2004, jun.). Psiquiatria e neurociências: como "redescobrir" o cérebro sem eclipsar o sujeito. Revista Latinoamericana de Psicopatologia Fundamental, $\operatorname{VII}(2), 110-124$.

Sisti, D. et al. (2013). Defining Mental Illnesses: Can Values and Objectivity Get Along? BMC Psychiatry, 13, 346-349.

\section{Resumos}

(The Research Domain Criteria project and the abandonment of the tradition of psychopathology)

This article explores the epistemological underpinnings of the Research Domain Criteria $(R D o C)$, launched by the National Institute of Mental Health (NIMH) - USA. The project has the objective of empirically investigating mental disorders in a biological framework. We discuss the sharp criticism received by the DSM shortly before its fifth edition came out, based as it was on the neuroscientific approach adopted by the RDoC. The preference for pathophysiology, combined with the exclusion of first-person experience, points to a scenario where research and clinical work are irrevocably divorced, and runs the risk of fostering an odd psychiatry with neither psyche nor pathos.

Key words: RDoC, classification systems, mental disorders, DSM, psychiatric clinics 
(Le projet Research Domain Criteria (RDoC) et le renoncement $\grave{a}$ la tradition psychopatologique)

Cet article examine les présupposés épistémologiques de l'initiative dénommée Research Domain Criteria (RDoC) proposée aux Etats-Unis par le National Institute of Mental Health (NIHM). Ce projet s'inscrit dans le cadre d'une approche des troubles mentaux ancrée dans une perspective essentielment biologique et empirique. Il faut remarquer que ce programme a été lancé à la veille de la presentation de la cinquième version du système DSM. On s'interroge si la mise en ouvre d'un tel projet, largement fondé sur l'axe de recherche neuroscientifique et physiopathologique - divorcée des toutes complexités de l'expérience vécue en première personne - ne donneront pas naissance à une étrange créature: la psychiatrie sans pathos et sans psyché.

Mots clés: RDoC, systèmes de classification, troubles mentaux, DSM, clinique psychiatrique

(El proyecto Research Domain Criteria y el abandono de la tradición psicopatológica)

El artículo examina los presupuestos epistemológicos del Research Domain Criteria (RDoC) - proyecto de investigación empírica de los transtornos mentales, dentro de una plataforma biológica - promovido pelo National Institute of Mental Health (NIMH). Discute el ataque dirigido al sistema DSM, poco antes de la publicación de su quinta edición, desde una perspectiva neurocientifica adoptada pelo RDoC. La apuesta por la fisiopatología, combinada con la exclusión de la experiencia descrita en la primera persona, apunta para un escenario en el cual investigación y clínica se divorciam con el riesgo del adviento de una psiquiatría extraña, sin psiquis y sin pathos.

Palabras claves: RDoC, sistemas de classificación, transtornos mentales, DSM, clinica psiquiátrica

(Das Projekt Research Domain Criteria und die Abwendung von der psychopathologischen Tradition)

Dieser Beitrag befasst sich mit den epistemologischen Voraussetzungen des Research Domain Criteria (RDoC) — einem empirischen Forschungsprojekt zu Geistesstörungen. Dies findet auf einer biologischen Plattform statt und wird vom National Institute of Mental Health (NIMH) gefördert. Hier wird der Angriff auf das DSM-System ausgehend von der neurowissenschaftlichen Perspektive des RDoC diskutiert, kurz vor der Herausgabe der 5. Auflage. Setzt man auf die Pathophysiologie in Kombination mit dem Ausschluss der in der ersten Person beschriebenen Erfahrung, ist ein Szenarium zu erkennen, in dem die Forschung und die Klinik sich scheiden und das Risiko besteht, dass eine fremde Psychiatrie ohne Psyche und ohne Pathos entsteht.

Schlüsselwörter: RDoC, Klassifizierungssysteme, Geistesstörungen, DSM, psychiatrische Klinik 


\section{OBSERVANDO A PSIQUIATRIA}

Citação/Citation: Zorzanelli, R., Dalgalarrondo, P., \& Banzato C. E. M. (2014, junho). O projeto Research Domain Criteria e o abandono da tradição psicopatológica. Revista Latinoamericana de Psicopatologia Fundamental, 17(2), 328-341.

Editor do artigo/Editor: Prof. Dr. Cláudio E. M. Banzato e Dra. Rafaela Zorzanelli

Recebido/Received: 16.4.2014/ 4.16.2014 Aceito/Accepted: 30.4.2014 / 4.30.2014

Copyright: (C) 2009 Associação Universitária de Pesquisa em Psicopatologia Fundamental/ University Association for Research in Fundamental Psychopathology. Este é um artigo de livre acesso, que permite uso irrestrito, distribuição e reprodução em qualquer meio, desde que o autor e a fonte sejam citados / This is an open-access article, which permits unrestricted use, distribution, and reproduction in any medium, provided the original author and source are credited.

Financiamento/Funding: Os autores declaram não ter sido financiados ou apoiados / The authors have no support or funding to report.

Conflito de interesses/Conflict of interest: Os autores declaram que não há conflito de interesses / The authors declare that has no conflict of interest.

\section{Rafaela Zorzanelli}

Psicóloga; Doutora em Saúde Coletiva; Professora Adjunta do Instituto de Medicina Social da Universidade do Estado do Rio de Janeiro - UERJ (Rio de Janeiro, RJ, Br).

Rua São Francisco Xavier, 524 - Pavilhão João Lyra Filho, $7^{\circ}$ andar/blocos D e E, e $6^{\circ}$ andar / bloco E - Maracanã

20550-013 Rio de Janeiro, RJ, Br

Fones: (21) 2334-0235 / 2334-0354

e-mail: rtzorzanelli@gmail.com

\section{Paulo Dalgalarrondo}

Psiquiatra; Doutor em Psiquiatria; Doutor em Antropologia; Professor Titular do Departamento de Psicologia Médica e Psiquiatria da Faculdade de Ciências Médicas da Universidade de Campinas - Unicamp (Campinas, SP, Br).

Rua Tessália Vieira de Camargo, 126 - Cidade Universitária "Zeferino Vaz"

13083-887 Campinas, SP, Br

Fone: (19) 3521-7206

e-mail: pdalga@fcm.unicamp.br

\section{Cláudio E. M. Banzato}

Psiquiatra; Doutor em Filosofia; Professor Associado do Departamento de Psicologia Médica e Psiquiatria da Faculdade de Ciências Médicas da Universidade de Campinas - Unicamp (Campinas, SP, Br).

Rua Tessália Vieira de Camargo, 126 - Cidade Universitária "Zeferino Vaz"

13083-887 Campinas, SP, Br

Fone: (19) 3521-7206

e-mail: cbanzato@fcm.unicamp.br 